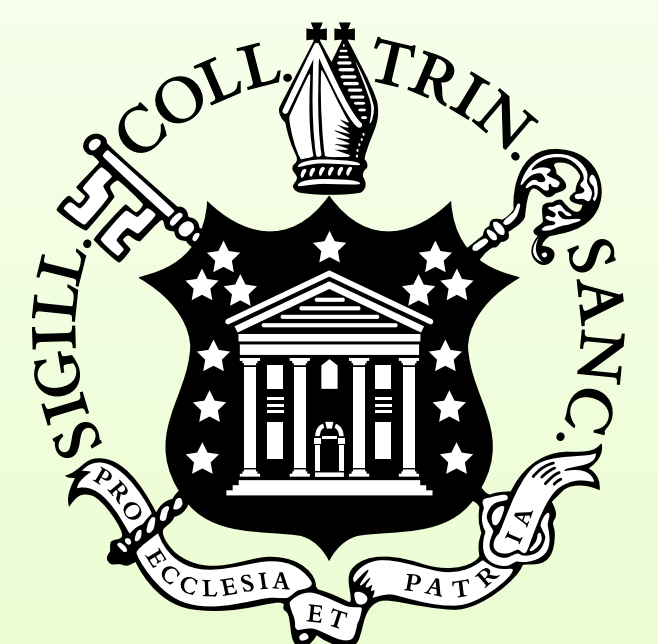

\title{
Trinity College
}

\section{Abstract}

Laplacian eigenmap is a useful technique to improve clusterbased segmentation of multivariate images. However, this approach requires an excessive amount of computations when processing large image datasets. To that end, we present a GPU-based acceleration procedure for vessel segmentation problems.

\section{Laplacian Eigenmap}

As described in Laskaris et. al. [1], the Laplacian Eigenmap is an effective dimensionality reduction method that maps a set of multivariate features vectors to points on the real line. Each features vector characterizes a group of neighboring pixels referred to as a patch. Once the projection is completed, a conventional method can be used to classify the projected points into one or more clusters.

(1) Generate patches - Let $\mathbf{x}_{i}$ be the multidimensional vector representing the $i^{\text {th }}$ patch.

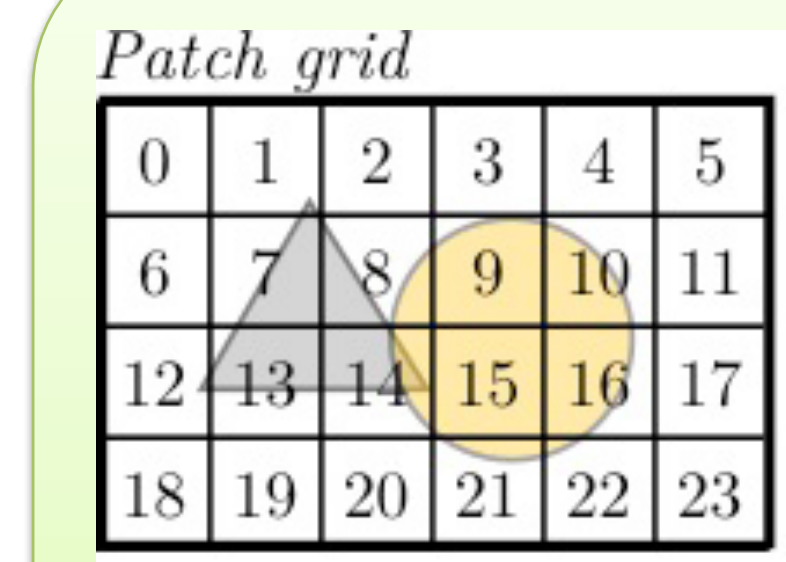

\section{Generate the weight matrix $W$}

\section{- Build a weighted} graph whose nodes are the terminals of the vectors. Let $W$ be the associated weight matrix.

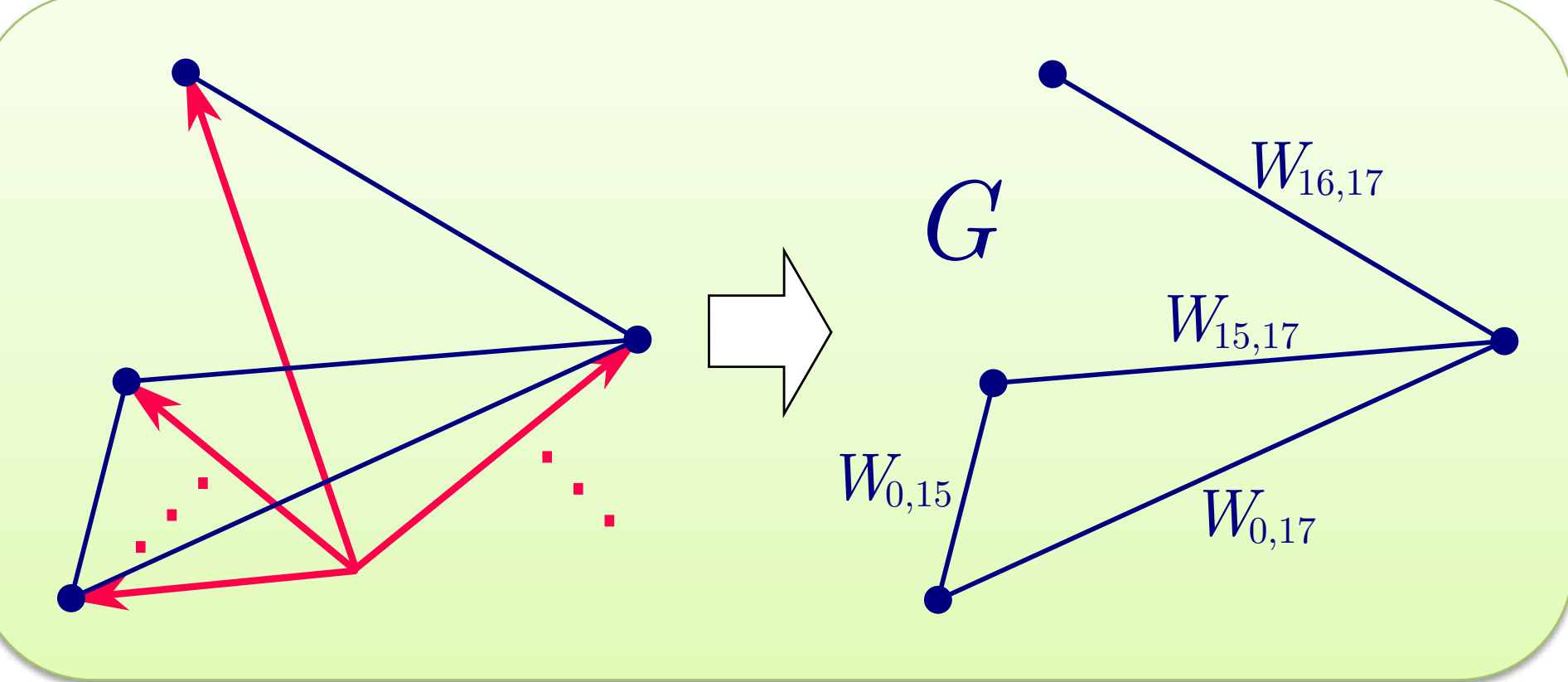

- Characteristics of $W$

- Two endpoints that are close enough should be connected with an edge. $\rightarrow W$ is symmetric

An edge carries more weight as its endpoints are far apart.

$$
W_{i j}=\exp \left(-\frac{\left\|\mathbf{x}_{i}-\mathbf{x}_{j}\right\|^{2}}{M \alpha^{2}}\right) \exp \left(-\frac{\left\|\mathbf{p}_{i}-\mathbf{p}_{j}\right\|^{2}}{2 \beta^{2}}\right)
$$

diff square()

Compute "difference vectors" $\mathbf{d}_{i j}$ and $\mathbf{f}_{\mathbf{i j}}$

$$
\mathbf{d}_{i j}(k)=\left(\mathbf{x}_{i}(k)-\mathbf{x}_{j}(k)\right)^{2}
$$$$
\mathbf{f}_{i j}(k)=\left(\mathbf{p}_{i}(k)-\mathbf{p}_{j}(k)\right)^{2}
$$

Divide labor among GPU blocks.

Fix index $j$ and vary $i$ from 0 to $N-1$, where $N$ is the number of features vectors.

There is no data dependency between any two difference vectors.
GPU Accelerated Vessel Segmentation Using Laplacian Eigenmaps

Lin Cheng, Hyunsu Cho, Peter Yoon, and Jiajia Zhao Trinity College, Hartford, CT 06106

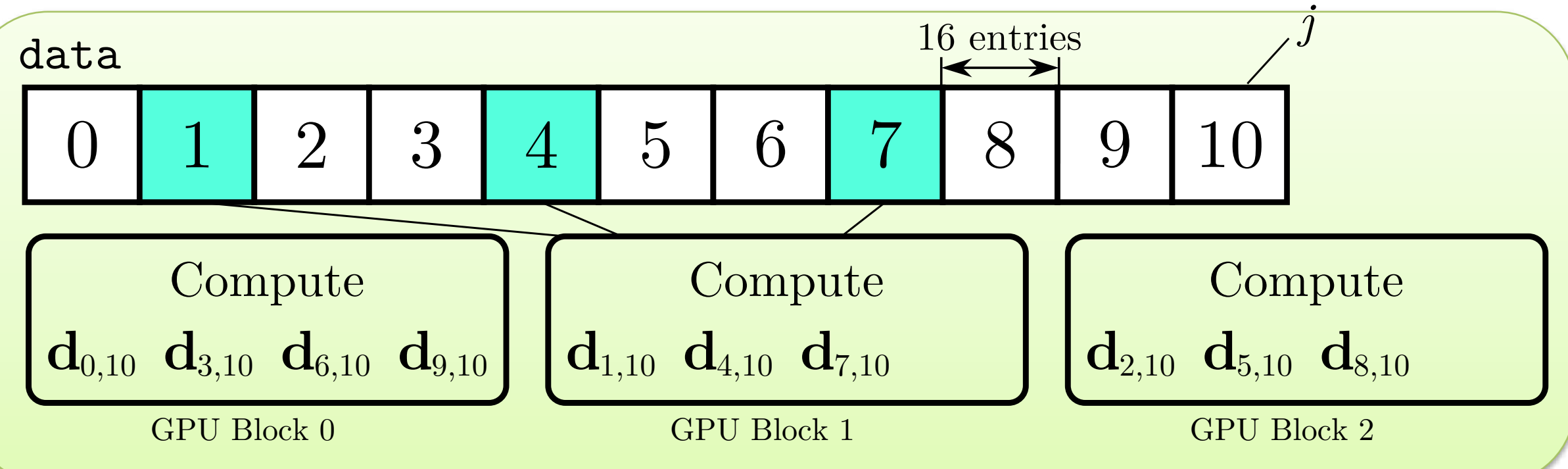

reduce (): binary reduction Use multiple GPU threads to compute partial sums in the shared memory.

Synchronization is required.

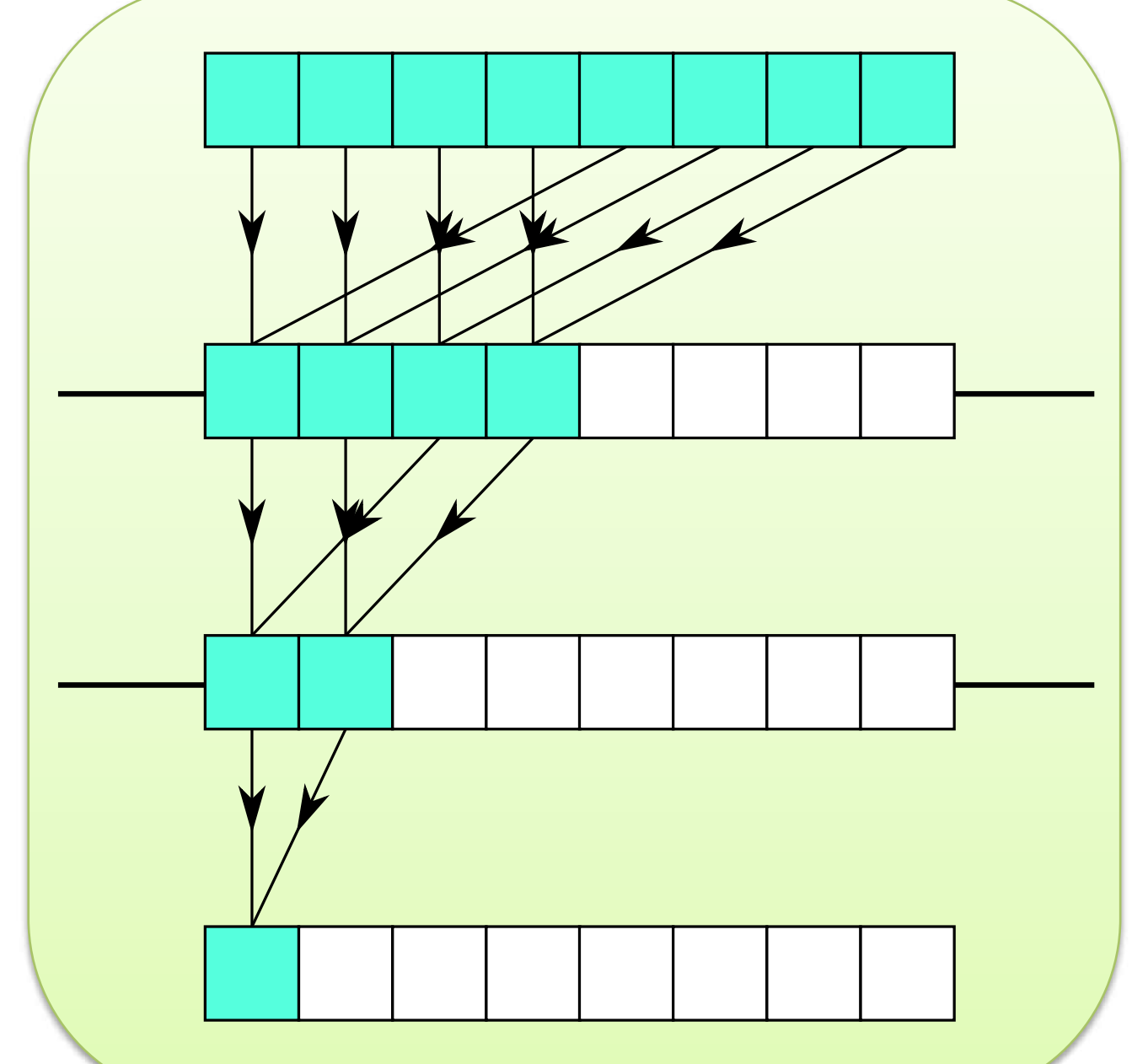

(3) Compute Laplacian matrix $L$ - $L=I-D^{1 / 2} W D^{1 / 2}$

$D$ is a diagonal matrix where each entry is the sum of the corresponding column of $W$.

- Use cublasDsymm() for a matrix multiplication.

4 Solve the generalized eigenvector problem

- A solution $\mathbf{y}=\left(y_{0}, y_{1}, \cdots, y_{N-1}\right)$ to the generalized eigenvector problem $L \mathbf{y}=\lambda D \mathbf{y}$ defines the map.

- Use magma_dsyevd( ) in MAGMA.

Compute all the eigenvalues and eigenvectors of a symmetric matrix.

- Components of $\mathbf{y}$ are the projections of the multivariate features vectors onto the real line.

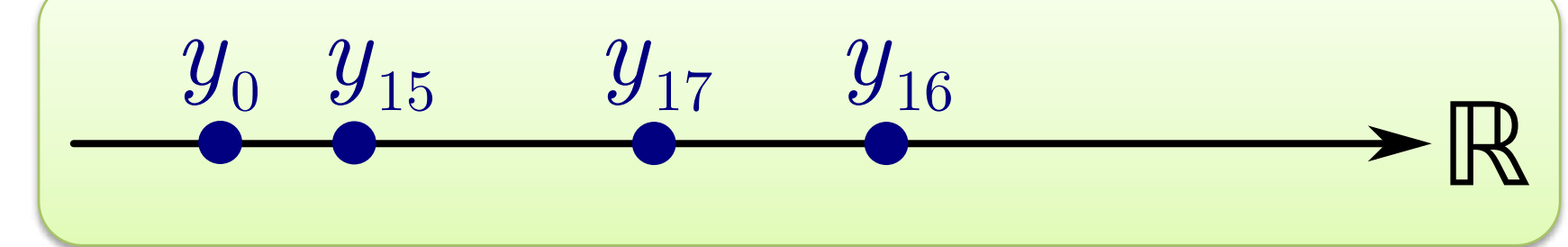

\section{Results and Performance}

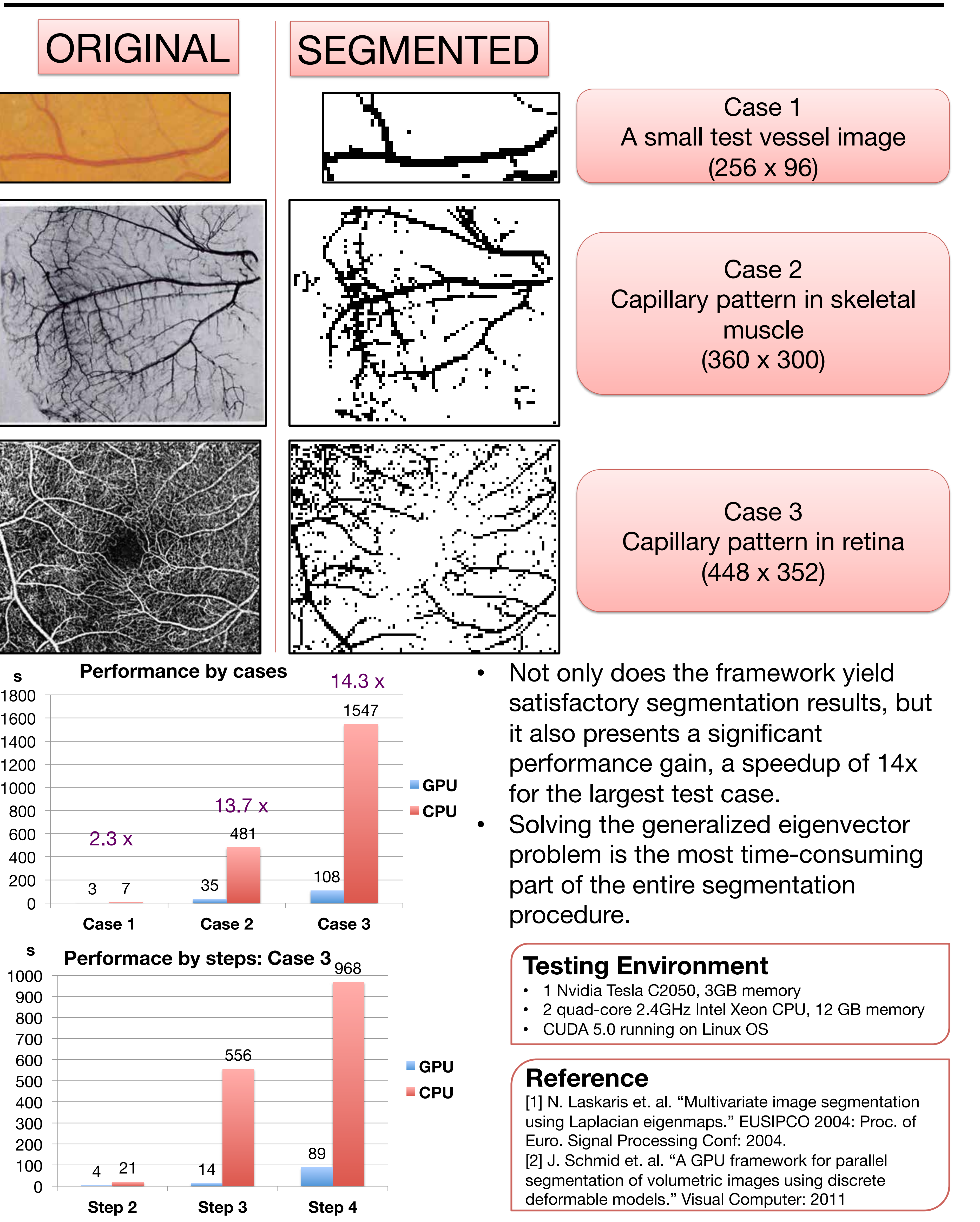

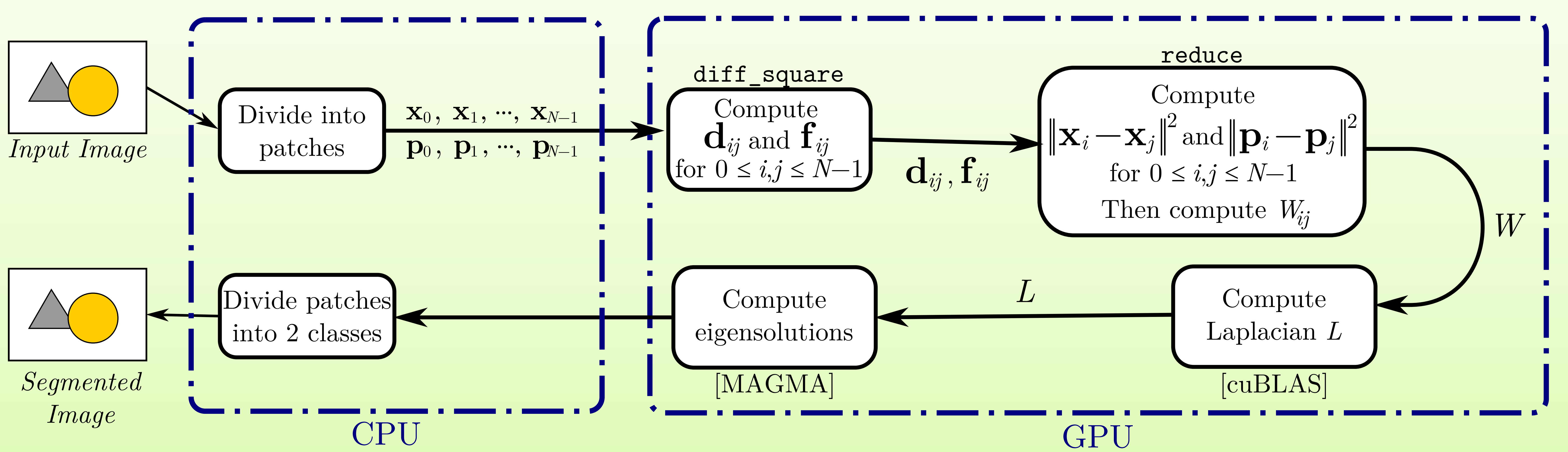

